$\mathrm{XX}$ Міжнародний симпозіум «Методи дискретних особливостей в задачах математичної фізики/Discrete Singularities Methods in Mathematical Physics»,

УДК 517.9

МДОЗМФ/DSMMPh-2021

MSC 74A99

\title{
Modeling of Finite Inhomogeneities by Discret Singularities
}

\author{
G.M. Zrazhevsky, V.F. Zrazhevska \\ Taras Shevchenko National University of Kyiv, Kyiv, Ukraine \\ National Technical University of Ukraine "Igor Sikorsky Kyiv Polytechnic Institute", \\ Kyiv, Ukraine \\ E-mail: zgrig@univ.kiev.ua
}

\begin{abstract}
This work focuses on development of a mathematical apparatus that allows to perform an approximate description of inhomogeneities of finite sizes in a continuous bodies by arranging the sources given on sets of smaller dimensions. The structure and properties of source densities determine the adequacy of the model. The theory of differential forms and generalized functions underlies this study. The boundary value problems with nonsmooth coefficients are formulated. The solutions of such problems is sought in the form of weakly convergent series and as an alternative - an equivalent recurrent set of boundary value problems with jumps. A feature of this approach is the ability to consistently improve the adequacy of the description of inhomogeneity. This is important because it allows to qualitatively assess the impact of real characteristic properties on the accuracy of the model description. Reducing the dimensions of inhomogeneities allows the use of efficient methods such as the Green's function and boundary integral equations to obtain a semi-analytic solution for direct and inverse problems. The work is based on a number of partial problems that demonstrate the proposed approach in modeling of inhomogeneities. The problems of modeling of the set of finite defects in an oscillating elastic beam, the set of inhomogeneities of an arbitrary shape in an oscillating plate, fragile cracks in a twodimensional elastic body under static loading are considered.
\end{abstract}

Key words: modeling of inhomogeneities, generalized functions, discret singularits, boundary value problems with nonsmooth coefficients.

\section{Моделювання скінченних неоднорідностей дискретними особливостями}

\section{Г.М. Зражевський, В.Ф. Зражевська}

Київський національний університет імені Тараса Шевченка, Київ, Україна, Національний технічний університет України "Київський політехнічний інститут імені Ігоря Сікорського", Київ, Україна

E-mail: zgrig@univ.kiev.ua

Робота присвячена розробці математичного апарату, що дозволяє виконувати наближений опис неоднорідностей скінченних розмірів в суцільному середовищі шляхом розташування джерел, заданих на множинах меншої розмірності. Структура та властивості густин джерел визначають адекватність моделі неоднорідності. Теорія диференціальних форм та узагальнених функцій лежить в основі даного дослідження. В роботі формулюються граничні задачі з негладкими коефіцієнтами. Розв'язок таких задач розшукується у вигляді слабко збіжних рядів та, як альтернатива, еквівалентного рекурентного набору граничних задач зі стрибками. Особливістю даного підходу є можливість послідовного покращення адекватності опису неоднорідності. Це є важливим, оскільки дозволяє якісно оцінити вплив реальних характерних властивостей неоднорідностей на точність опису моделі. Зменшення розмірностей неоднорідності дозволяє використовувати ефективні методи типу мето-

() Зражевський Г.М., Зражевська В.Ф., 2021 


\begin{abstract}
дів функції Гріна та граничних інтегральних рівнянь для отримання напіваналітичного розв'язку для прямих та обернених задач. Робота побудована на ряді часткових задач, що демонструють запропонований підхід при моделюванні неоднорідностей. Розглянуті наступні задачі: моделювання сукупності скінченних дефектів в пружній балці, що коливається, моделювання сукупності неонорідностей довільної форми в платівці, моделювання крихких тріщин в двовимірному пружному тілі при статичному навантаженні.
\end{abstract}

Ключові слова: моделювання неоднорідностей, узагальнені функції, точкові особливості, граничні задачі з негладкими коефіцієнтами.

\title{
1. Вступ
}

Побудові моделей пружних тіл з неоднорідними включеннями, що мають фізичні характеристики, відмінні від характеристик основного матеріалу, присвячена велика кількість наукових та науково-інженерних досліджень. Це пояснюється практичною значимістю тематики. В техніці є актуальним розрахунок конструкцій, складених з елементів з різними механічними властивостями, або такими, що їх природній опис передбачає різну геометричну розмірність [1-5]. В дефектоскопії та акустичному скануванні основною задачею $\epsilon$ визначення фізичних та геометричних характеристик $[4,6]$. При керуванні динамічною поведінкою коливних та хвильових процесів в пружних тілах важливе значення має розрахунок відгуку 3 врахуванням наявності неоднорідностей з невідомими характеристиками [7]. Звичайно, це не є повний перелік практичних галузей, що потребують моделювання неоднорідностей в пружних тілах. Найбільш поширеними в науковій літературі $\epsilon$ дослідження стрибкоподібної зміни густини та характеристик пружності. В багатьох випадках опис включення потребує зміни моделі поведінки, як, наприклад, у випадку дослідження рівноваги тіл з тріщинами, ансамблями дислокацій чи зонами пластичної течії $[4,5]$. Зміна характеристик (в загальному сенсі розуміння цього терміну) може прийматись як детермінованою, так і випадковою. Тобто, опис включень $є$ багатофакторним та на цей час не може бути зведений до єдиної універсальної математичної процедури. Цим пояснюється значне різноманіття як технік математичного моделювання, так і способів та методів математичного опису. Широко вживаними є метод часткових областей $[8,9]$, граничних інтегральних рівнянь [9], варіаційні методи. Слід відмітити також широке використання апарату узагальнених функцій при моделюванні скінченних неоднорідностей різної природи $[8,9]$.

В роботі пропонується проста математична техніка використання апарату диференціальних форм в теорії узагальнених функцій [10], що застосовується до розв'язання граничних задач з негладкими коефіцієнтами. Техніка дозволяє моделювати скінченні неоднорідності шляхом зменшення їхньої геометричної розмірності, зокрема, до точкових сингулярностей. Для випадку відомих задач, наприклад, моделювання сейсмічного джерела еквівалентною точковою сингулярністю із заданням тензору сейсмічного моменту [9], запропонована техніка призводить до тих же результатів.

В багатьох задачах рівняння рівноваги субфізичного елементу має вигляд:

$$
\mathrm{LD} \vec{\varphi}+\overrightarrow{\mathrm{X}}=0
$$


XX Міжнародний симпозіум «Методи дискретних особливостей в задачах математичної фізики/Discrete Singularities Methods in Mathematical Physics»,

МДОЗМФ/DSMМРh-2021

де $\mathrm{L}$ - лінійний диференціальний оператор, D - оператор, що визначається моделлю та рівняннями стану, який може бути нелінійним та містити негладкі коефіцієнти, $\overrightarrow{\mathrm{X}}$ - узагальнене навантаження. Легко бачити, що (1) може бути зведено в загальному вигляді до рівняння виду:

$$
\mathrm{L} \vec{\sigma}=-\overrightarrow{\mathrm{X}}+\mathrm{L}(\mathrm{E} \vec{\sigma}), \vec{\sigma}=S \vec{\varphi},
$$

в якому права частина містить узагальнені функції.

Продемонструємо застосування запропонованого підходу до задач механіки деформівного твердого тіла.

2. Функція Гріна для задачі гармонійних коливань балки Бернуллі зі скінченним включенням

Функція Гріна $w(x, \zeta)$ для задачі про гармонійні коливання шарнірно закріпленої балки Бернуллі зі скінченним включенням, що розташовується в області $|x-\chi|<\ell$ та містить матеріал зі зміненим на $\Delta E$ модулем Юнга, визначається граничною задачею:

$$
\begin{aligned}
& \frac{d^{2}}{d x^{2}}\left(E(x) I \frac{d^{2} w}{d x^{2}}\right)-\omega^{2} \rho w=\delta(x-\xi), x \in(0,1) \\
& \quad w(0 ; \zeta)=w(1 ; \zeta)=0, d^{2} w(x ; \zeta) /\left.d x^{2}\right|_{x=0}=d^{2} w(x ; \zeta) /\left.d x^{2}\right|_{x=1}=0,
\end{aligned}
$$

де $E(x)=E_{0}(1-\Delta E f(x)), f(x)=H(x-(\chi-\ell))-H(x-(\chi+\ell))$.

Згідно з (1), (2) представимо (3) у вигляді:

$$
I E(x) \frac{d^{4} w}{d x^{4}}-\omega^{2} \rho w=\delta(x-\xi)-I \frac{d^{2} E}{d x^{2}} \frac{d^{2} w}{d x^{2}}-2 I \frac{d E}{d x} \frac{d^{3} w}{d x^{3}} .
$$

Скориставшись розкладами $f^{\prime}=2 \sum_{k=0}^{\infty} \frac{\delta^{(2 k+1)}(x-\chi)}{(2 k+1) !} \ell^{2 k+1}$ i $f^{\prime \prime}=2 \sum_{k=0}^{\infty} \frac{\delta^{(2 k+2)}(x-\chi)}{(2 k+1) !} \ell^{2 k+1}$, рівняння (3) можемо представити у вигляді:

$$
\begin{aligned}
& (1-\Delta E f(x)) w_{x}^{(4)}(x, \xi)-\pi^{4} k^{4} w(x, \xi)=\delta(x-\xi)+ \\
& 2 \Delta E w_{x}^{\prime \prime}(x, \xi) \sum_{k=0}^{\infty} \frac{\delta^{(2 k+1)}(x-\chi)}{(2 k+1) !} \ell^{2 k+1}+4 \Delta E w_{x}^{(3)}(x, \xi) \sum_{k=0}^{\infty} \frac{\delta^{(2 k+2)}(x-\chi)}{(2 k+1) !} \ell^{2 k+1} .
\end{aligned}
$$

Розв'язок (4) шукаємо у вигляді $w=w_{0}+\hat{w}, \hat{w}=\bar{o}\left(w_{0}\right)$. При виконанні умови $\ell \ll 1$ має місце асимптотичний розклад:

$$
\hat{w}=\varepsilon_{1} w_{1}+\varepsilon_{2} w_{2}+\ldots
$$

де $\varepsilon_{i+1}=\bar{o}\left(\varepsilon_{i}\right)$. Підстановка $\hat{w}$ в (4) дозволяє отримати $\varepsilon_{i}=\frac{2 \ell^{2 i-1} \Delta E}{(2 i-1) !}, i=1,2, \ldots$, a, отже, рівняння (4) еквівалентне набору рівнянь:

$$
\left\{\begin{array}{rl}
w_{0 x}^{(4)}-\pi^{4} k^{4} w_{0}= & \delta(x-\xi), \\
w_{i x}^{(4)}-\pi^{4} k^{4} w_{i}= & w_{0 x}^{(4)} \delta^{(2 i-2)}(x-\chi)+2 w_{0 x}^{(3)} \delta^{(2 i-1)}(x-\chi)+ \\
& +w_{0 x}^{(2)} \delta^{(2 i)}(x-\chi),
\end{array} \quad i=1,2, \ldots .\right.
$$

Праві частини (5) можна спростити, використавши очевидні тотожності типу: 


$$
\frac{d^{2}}{d x^{2}}\left(\delta^{(2 i-2)}(x-\chi) w_{0 x}^{(2)}\right)==\left.\sum_{s=0}^{2 i-2} C_{2 i-2}^{s} \frac{d^{2 i-s}}{d x^{2 i-s}} w_{0}(x, \zeta)\right|_{\mathrm{x}=\chi} \delta^{(2+s)}(x-\chi) .
$$

Скориставшись (5) можна легко побудувати наближення як завгодно високого порядку. Так, перше наближення знаходиться з граничної задачі:

$$
\begin{aligned}
& w_{1 x}^{(4)}-\pi^{4} k^{4} w_{1}=w_{0 x}^{(2)}(\chi, \xi) \delta^{\prime \prime}(x-\chi) \\
& \left\{\begin{array}{l}
w_{1}(0, \xi, \chi)=w_{1}(1, \xi, \chi)=0 \\
w_{1 x}^{(2)}(0, \xi, \chi)=w_{1 x}^{(2)}(1, \xi, \chi)=0
\end{array}\right.
\end{aligned}
$$

де $w_{0}(x, \zeta)$ - функція Гріна для однорідної балки, отже, розв'язок (6) має вигляд:

$$
w_{1}=\frac{\partial^{2} w_{0}(\chi, \zeta)}{\partial x^{2}} \frac{\partial^{2} w_{0}(x, \chi)}{\partial \zeta^{2}}
$$

При необхідності можна побудувати наближення довільного порядку.

Отже, мала неоднорідність може бути змодельована точковим джерелом, а функція Гріна побудована у вигляді слабко збіжного ряду. Маніпулювання коефіцієнтами при сингулярностях в правій частині (5) дозволяє зробити модель неоднорідності багатопараметричною.

3. Гармонійні коливання платівки зі скінченним включенням.

Гармонійні коливання в платівці $з$ циліндричною жорсткістю $D_{0}$ та густиною $\rho_{0}$ (за моделлю Кірхгофа), що містить неоднорідність в області $P(x, y)>0$ з циліндричною жорсткістю $D_{1}$ та густиною $\rho_{1}$, під дією навантаження $q$ описуються рівняннями:

$$
\left\{\begin{array}{cc}
\mathbf{L}\left(-D_{0} \mathbf{L}_{1}\right) \mathbf{L}_{2} w+\rho_{0} \omega^{2} w+q=0, & P(x, y)<0 \cap \Omega \\
\mathbf{L}\left(-D_{1} \mathbf{L}_{1}\right) \mathbf{L}_{2} w+\rho_{1} \omega^{2} w=0, & P(x, y)>0
\end{array},\right.
$$

де $\mathrm{L}=\left[\frac{\partial^{2}}{\partial x^{2}} \frac{\partial^{2}}{\partial y^{2}} \frac{\partial^{2}}{\partial x \partial y}\right], \mathrm{L}_{1}=\left[\frac{\partial}{\partial x} \frac{\partial}{\partial y}\right], \mathrm{L}_{2}: \vec{M}=-D \mathrm{~L}_{2} w$.

Вважаємо, що $\vec{\nabla} P_{P=0} \neq 0$, область $P$ - однозв'язна та опукла. Ввівши позначення: $D_{1}=D_{0}-\Delta D, \frac{\Delta D}{D_{0}}=\kappa_{D}, \frac{\rho_{0} \omega^{2}}{D_{0}}=k^{4}, \kappa_{\rho}=\frac{\rho_{0}-\rho_{1}}{\rho_{0}}, \theta(P)=\left\{\begin{array}{ll}1, & P>0 \\ 0, & P<0\end{array}\right.$ (функція області), (8), згідно з (1), (2), можна записати у вигляді:

$$
\mathbf{L L}_{1} \mathbf{L}_{2} w-k^{4} w=\frac{q}{D_{0}}+\kappa_{D} \mathbf{L} \theta(P) \mathbf{L}_{1} \mathbf{L}_{2} w-k^{4} \kappa_{P} \theta w,(x, y) \in \Omega .
$$

Знехтувавши в (9) зміною густини та позначивши $\vec{v}==\mathbf{L}_{1} \mathbf{L}_{2} w$, розглянемо сингулярний член правої частини. Очевидно, що $\langle\mathbf{L} \vec{v} \theta, \Phi\rangle=\langle\vec{v} \theta, \mathbf{L} \Phi\rangle$, де $\Phi-$ функція 3 основного простору.

Згідно $з$ теоремою про середнє: $\langle\vec{v}, \mathbf{L} \Phi\rangle=\left.S_{\rho} \vec{v}(X)\left(\frac{\partial^{2} \Phi}{\partial x^{2}}, 2 \frac{\partial^{2} \Phi}{\partial x \partial y}, \frac{\partial^{2} \Phi}{\partial y^{2}}\right)\right|_{X}+\underline{\underline{O}}\left(S_{\rho}{ }^{2}\right)$ де $S_{\rho}=\operatorname{pow}(P)$, а $X=(X, Y)$ - центральна точка області $P$. Тоді: 
XX Міжнародний симпозіум «Методи дискретних особливостей в задачах математичної фізики/Discrete Singularities Methods in Mathematical Physics», МДОЗМФ/DSMМPh-2021

$$
\begin{aligned}
& \mathbf{L} \theta(P) \vec{v}=S_{\rho} \vec{v}\left(\frac{\partial^{2} \delta(x-X)}{\partial x^{2}} \delta(y-Y), 2 \frac{\partial \delta(x-X)}{\partial x} \frac{\partial \delta(y-Y)}{\partial y}, \frac{\partial^{2} \delta(y-Y)}{\partial y^{2}} \delta(x-X)\right)+ \\
& \quad+\underline{\underline{O}}\left(S_{\rho}{ }^{2}\right)
\end{aligned}
$$

Вираз (10) визначає дискретне перше наближення впливу неоднорідного включення, що має вигляд зрівноважених точкових диполів. Як бачимо, перше наближення не залежить від форми дефекту та має інтенсивність, що пропорційна площі області неоднорідності. Це є цілком очікуваним з огляду на відому теорему:

$$
F(P)=\iint_{P \geq 0} f(x, y) d x d y \Rightarrow \frac{d F}{d S}=f(x, y),\left(S=\iint_{P \geq 0} d x d y\right)
$$

Модель $\epsilon$ трипараметричною, оскільки визначається вектором $\vec{v}$. В найпростішому випадку $\vec{v}$ може бути визначений через згинаючий момент в уявній однорідній платівці в точці розташування неоднорідності.

4. Моделювання крихкої тріщини в статичній теорії пружності.

Рівняння статичної рівноваги пружної області $\Omega$ в умовах плоского напруженого стану має вигляд:

$$
\begin{aligned}
& \mathbf{L}^{T} \mathrm{D} \mathbf{L} \vec{u}+\overrightarrow{\mathrm{X}}=0, \quad(x, y) \in \Omega, \\
& \text { де } \mathbf{L}=\left[\begin{array}{cc}
\frac{\partial}{\partial x} & 0 \\
0 & \frac{\partial}{\partial y} \\
\frac{\partial}{\partial y} & \frac{\partial}{\partial x}
\end{array}\right], \mathrm{D}=\frac{E}{1-v^{2}}\left[\begin{array}{ccc}
1 & v & 0 \\
v & 1 & 0 \\
0 & 0 & \frac{1-v}{2}
\end{array}\right], \vec{\varepsilon}=\left[\begin{array}{l}
\varepsilon_{x} \\
\varepsilon_{y} \\
\varepsilon_{x y}
\end{array}\right]=\mathbf{L} \vec{\varphi}, \vec{\sigma}=\left[\begin{array}{l}
\sigma_{x} \\
\sigma_{y} \\
\sigma_{x y}
\end{array}\right]=\mathrm{D} \vec{\varepsilon} .
\end{aligned}
$$

Нехай в області $\Omega \in$ крихка тріщина, що розташована на розімкненому, скінченному відрізку кривої $P(x, y)=0 \quad$ такому, що $\quad|\vec{\nabla} P|_{P=0} \mid=1, \quad$ а $\hat{v}^{T}=\left(\alpha_{x}, \alpha_{y}\right), \hat{\tau}^{T}=\left(-\alpha_{y}, \alpha_{x}\right)$ - відповідно нормаль та дотична до тріщини $(\hat{v}=\vec{\nabla} P)$.

Задамо на тріщині скінченний стрибок переміщень: $[\vec{u}]=\hat{n}\left[u_{n}\right]+\hat{\tau}\left[u_{\tau}\right]$. Компоненти тензору напружень будуть мати сингулярні частини. Приймаючи до уваги, що $\frac{\partial \delta(P)}{\partial \tau}=0, \frac{\partial \delta(P)}{\partial n}=\delta^{\prime}(P), \frac{\partial\left[u_{n}\right]}{\partial n}=0, \frac{\partial\left[u_{\tau}\right]}{\partial n}=0$, компоненти вектора напружень $\vec{t}^{S}=t_{n}{ }^{S} \hat{n}+t_{\tau}{ }^{S} \hat{\tau}, \vec{q}^{S}=q_{n}{ }^{S} \hat{n}+t_{r}{ }^{S} \hat{\tau}$, в локальній системі координат $(\hat{\tau}, \hat{n})$ мають вигляд:

$$
\left\{\begin{array}{c}
t_{n}{ }^{S}=\frac{E}{1-v^{2}}\left[u_{n}\right] \delta(P) \\
t_{\tau}{ }^{S}=\frac{1-v}{2} \frac{E}{1-v^{2}}\left[u_{\tau}\right] \delta(P)
\end{array},\left\{\begin{array}{c}
q_{n}{ }^{S}=\frac{E}{1-v^{2}}\left[u_{\tau}\right] \delta(P) \\
q_{\tau}{ }^{S}=\frac{E}{1-v^{2}} \gamma\left[u_{\tau}\right] \delta(P)
\end{array}\right.\right.
$$

А, отже, еквівалентні об’ємні джерела, розподілені по відрізку тріщини, мають вигляд: 


$$
\left\{\begin{array}{c}
P_{n}^{S}=\left(\left[u_{n}\right] \delta^{\prime}(P)+\frac{(1-v)}{2} \frac{\partial}{\partial \tau}\left[u_{\tau}\right] \delta(P)\right) \frac{E}{1-v^{2}}, \\
P_{\tau}^{S}=\left(\frac{(1-v)}{2}\left[u_{\tau}\right] \delta^{\prime}(P)+v \frac{\partial}{\partial \tau}\left[u_{n}\right] \delta(P)\right) \frac{E}{1-v^{2}} .
\end{array}\right.
$$

Приймаючи до уваги, що

$$
\left\langle\frac{\partial}{\partial \tau}\left[u_{n}\right] \delta(P), \Phi\right\rangle=\left\langle\frac{\partial}{\partial \tau}\left(\delta(P)\left[u_{\tau}\right]\right), \Phi\right\rangle,
$$

(13) повністю збігається з відомим результатом [11].

\section{ЛІТЕРАТУРА}

1. Clouet E., Varvenne C., Jourdan T. Elastic modeling of point-defects and their interaction. Computational Materials Science. 2018, 147, pp.49-63.

2. Khludnev A.M. On thin inclusions in elastic bodies with defects. Z. Angew. Math. Phys. 70, 45. 2019. https://doi.org/10.1007/s00033-019-1091-5

3. Itou H., Khludnev A.M. On delaminated thin Timoshenko inclusion inside elastic bodies. Math. Methods Appl. Sci. 39. 2016, 17 4980-4993 pp.

4. Rubio L., Fernández-Sáez J., Morassi A. The full nonlinear crack detection problem in uniform vibrating rods. Journal of Sound and Vibration. 2015, 339, 99111.

5. Eshelby J.D. The elastic field outside an ellipsoidal inclusion. Proc. Roy. Soc. Lond. A 252, 1959, 561-569. doi:10.1098/rspa.1959.0173

6. Zrazhevsky G.M., Zrazhevska V.F. The Extension Method for Solving Boundary Value problem of the Theory of Oscillations of Bodies with Heterogeneity. 2020/3/22, World Journale of Engineering Research and Technology. Vol. 6, Iss. 2, pp. 503-514.

7. Zrazhevsky G.M., Golodnikov A.N., Uryasev S.P., Zrazhevsky A.G. Application of Buffered Probability of Exceedance in Reliability Optimization Problems. Cybernetics and Systems Analysis. 2020. Vol. 56, pp. 476-484.

8. Zrazhevsky G.M., Zrazhevska V.F. Usage of generalized functions formalism in modeling of defects by point singularity. Вісник Київського національного університету імені Тараса Шевченка. Серія: фізико-математичні науки. 2019. Iss. 1, pp. 58-61.

9. Aki K., Richards P. Quantitative Seismology, 2nd Ed. University Science Books, 2002.

10. Gelfand I.M. Generalized Functions, Vol. 1, AMS Chelsea Publishing, 1964.

11. Crouch S.L., Starfield A.M. Boundary Element Methods in Solid Mechanics. George Allen \& UNWIN, 1983. London. 
XX Міжнародний симпозіум «Методи дискретних особливостей в задачах математичної фізики/Discrete Singularities Methods in Mathematical Physics»,

\section{МДОЗМФ/DSMMPh-2021}

\section{Моделирование конечных неоднородностей дискретными особенностями}

\section{Г.М. Зражевский, В.Ф. Зражевская}

Киевский национальный университет имени Тараса Шевченко, Киев, Украина, Национальный технический университет Украины "Киевский политехнический институт имени Игоря Сикорского", Киев, Украина E-mail: zgrig@univ.kiev.ua

Работа посвящена разработке математического аппарата, позволяющего приближенно описывать неоднородности конечных размеров в сплошной среде путем расположения источников, заданных на множествах меньшей размерности. Структура и свойства плотностей источников определяют адекватность модели неоднородности. Теория дифференциальных форм и обобщенных функций лежит в основе данного исследования. В работе формулируются граничные задачи с негладкими коэффициентами. Решение таких задач разыскивается в виде слабо сходящихся рядов и как альтернатива - эквивалентного рекуррентного набора граничных задач со скачками. Особенностью данного подхода является возможность последовательного улучшения адекватности описания неоднородности. Это важно, поскольку позволяет качественно оценить влияние реальных характерных свойств неоднородностей на точность описания модели. Уменьшение размерностей неоднородностей позволяет использовать методы типа функции Грина и граничных интегральных уравнений для получения полуаналитического решения прямых и обратных задач. Работа построена на ряде частных задач, демонстрирующих предложенный подход при моделировании неоднородностей. Рассмотрены задачи моделирования совокупности конечных дефектов в колеблящейся упругой балке, совокупности неоднородностей произвольной формы в пластинке, хрупких трещин в двумерном упругом теле при статическом нагружении.

Ключевые слова: моделирование неоднородностей, обобщенные функции, точечные особенности, граничные задачи с негладкими коэффициентами. 